\title{
A GESTÃO DA ENERGIA SOLAR FOTOVOLTAICA SOB A ÓTICA DA BIBLIOMETRIA
}

\author{
Carmen Brum Rosa ${ }^{2}$, Julio Cezar Mairesse Siluk ${ }^{1}$, Leandro Michels ${ }^{2}$, Paula Donaduzzi Rigo ${ }^{1}$, Graciele Rediske ${ }^{1}$ \\ 1 Programa de Pós-Graduação de Engenharia de Produção, Universidade Federal de Santa Maria, 97105-900, Santa Maria, Brasil. \\ 2 Programa de Pós-Graduação em Engenharia Elétrica,, Universidade Federal de Santa Maria, 97105-900, Santa Maria, Brasil.
}

*E-mail: carmenbrosa@gmail.com

Recebido em: $27 / 04 / 2018$

Aceito em: 03/05/2018

\section{RESUMO}

A geração de energia solar fotovoltaica cresce exponencialmente em todo o mundo, e esse crescimento técnico e comercial deve estar apoiado a estudos de gestão da energia através de ferramentas de apoio à tomada de decisão. Neste sentido, este artigo investigou, a partir de uma análise bibliométrica, o cenário atual das pesquisas acadêmicas na área de Energia Solar Fotovoltaica em cruzamento com os conceitos de Avaliação de Desempenho, Indicadores de Desempenho e Análise Multicritério de Decisão, em um período de 5 anos, de 2011 a 2016. Através desta busca, foram encontrados 49 artigos e constatou-se o potencial internacional dos estudos em energia fotovoltaica, confirmando que as pesquisas abordadas possuem um alto grau de impacto e de citações envolvendo diversos países. Porém, não foram encontrados estudos que envolvessem a temática de desenvolvimento de técnicas de gestão para esta fonte de energia que se encontra em constante expansão. Isso confirma uma lacuna no ambiente de pesquisa científica que deve ser preenchida, visto que estudos nesta área são essenciais para assegurar a continuidade do crescimento da energia solar fotovoltaica no mundo.

Palavras-chave: Energia Fotovoltaica. Gestão de Energia. Bibliometria

\section{Introdução}

A crescente demanda por eletricidade exigiu investimentos em novas fontes de energia para atender o rápido aumento do consumo e minimizar o impacto da crise energética. Nas últimas décadas, a geração de eletricidade por energia fotovoltaica e energia eólica recebeu atenção considerável em todo o mundo [1]. A maioria dos países está investindo no estudo de novas fontes de energia que podem ser conectadas ao sistema de distribuição para diversificar a matriz e evitar cenários de crise energética. Assim, de um ponto de vista estratégico, a energia solar fotovoltaica vem se destacando internacionalmente devido ao aumento do número de instalações, especialmente de sistemas solares montados no solo e em larga escala [2].

A pesquisa e o desenvolvimento de recursos energéticos alternativos sempre atraíram atenção significativa devido à sua fonte abundante e sustentável, no entanto, as incertezas associadas aos custos, ao armazenamento de energia e ao gerenciamento necessário para a implementação desses sistemas requerem uma sólida metodologia de gestão para tomada de decisão [3]. Diante deste cenário, para obter uma vantagem competitiva e defender posições estratégicas no mercado energético é necessário que os gestores conheçam e compreendam a dinâmica dos atores nos âmbitos empresarial e estrutural, permeados pelos indicadores capazes de mensurar, de fato, sua realidade específica. Além disso, torna-se determinante para o avanço deste setor o monitoramento da competitividade sistêmica, a qual se evidencia como sendo a barreira mais crítica para o desenvolvimento e para a ampliação desta fonte geradora de eletricidade. Sendo assim, percebe-se que a geração de energia fotovoltaica está inserida em um ambiente mundialmente competitivo, principalmente por apresentar-se em um setor com características específicas, o que obriga as unidades de geração de energia solar a desempenhar seus processos com máxima eficiência, principalmente no que diz respeito àqueles fatores considerados como os mais relevantes para a competitividade.

A partir dessa necessidade, verifica-se a importância da utilização de ferramentas gerenciais estruturadas e de metodologias de gestão que sejam capazes de auxiliar na identificação, mensuração e análise de indicadores, de modo a garantir posições competitivas no mercado da energia solar fotovoltaica [4]. Neste sentido, este estudo tem como objetivo investigar as características da literatura sobre conceitos de gerenciamento de energia solar compreendida entre os anos de 2011 a 2016 e sua implicação com as técnicas bibliométricas. Este método estatístico de análise bibliográfica avalia e quantifica o crescimento das publicações abordando um assunto em particular e examina as características de publicação de países, institutos de pesquisa e campos, revistas, autores, hábitos de citação e palavraschave do autor [5]. Além desse objetivo, o estudo tem como interesse analisar o progresso da pesquisa em gestão de energia solar fotovoltaica, evidenciando a importância da aplicação de 
técnicas e ferramentas da área de Engenharia de Produção e Administração de Empresas.

\section{Metodologia}

Quanto à estrutura dessa pesquisa, o trabalho foi dividido em três etapas sequenciais, sendo em um primeiro momento realizada uma retomada teórica sobre a metodologia alvo deste estudo. A bibliometria é uma ferramenta para analisar como as disciplinas evoluem com base na estrutura intelectual, estrutura social e estrutura conceitual [6]. Os autores ainda sugerem que os métodos bibliométricos são complementares dos métodos tradicionais de revisão e de revisões estruturadas da literatura e, quando comparados com esses métodos bibliométricos, aumentam a objetividade desses tipos de estudos. A bibliometria é utilizada para avaliar o desempenho da pesquisa e da publicação realizada por indivíduos, por instituições e pormapear a estrutura e a dinâmica da ciência [5].

As duas seguintes etapas foram realizadas conforme o enquadramento metodológico apresentado na Figura 1. No primeiro momento foram selecionadas as palavras-chave de acordo com a temática envolvida no estudo, que então foram submetidas a cruzamento para análise bibliométrica, esperando-se ao final apresentar as conclusões encontradas.

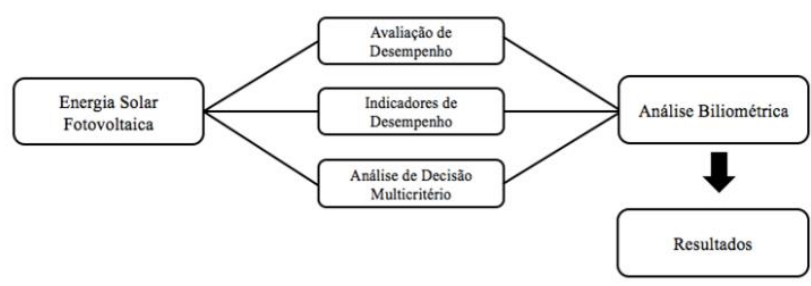

Figura 1- Enquadramento Metodológico.

Em específico para a etapa de cruzamento, a palavrachave "Energia Solar Fotovoltaica" se faz mais importante em função da pesquisa alvo envolver a geração de energia através da fonte fotovoltaica, para tanto, essa expressão se repete no cruzamento com as demais. Já, as palavras-chave "Avaliação de Desempenho", "Indicadores de Desempenho" e "Análise de Decisão Multicritério" são os conceitos de gestão a ser verificado o grau de relevância dentro dos estudos e aplicações de energia fotovoltaica nas estruturas intelectual, cultural e social, conforme evidencia a base da bibliometria. A coleta dos dados deu-se nas plataformas Science Direct, Scopus, Portal de periódicos da Coordenação de Aperfeiçoamento de Pessoal de Nível Superior (CAPES) e Emerald, entre o período fechado de janeiro de 2011 a agosto de 2016. As palavras-chave foram traduzidas para língua inglesa e inseridas no modo de busca avançado de cada plataforma, a fim de especificar e garantir o retorno apenas de artigos, sendo estes publicados na faixa de período selecionada e que continham a palavra-chave 1 e a palavra-chave 2 no seu título, resumo e/ou palavras-chave.

Após a etapa de cruzamento, as informações levantadas serviram de suporte para a verificação da frequência de pesquisa e de publicações na área de gestão de energia fotovoltaica, identificação de instituições, mapeamento da estrutura e a dinâmica da ciência. Os resultados permitiram a conclusão deste trabalho, conforme apresentado nas seções seguintes.

\section{Aplicação da Metodologia}

Através da implementação da metodologia, obteve-se um retorno de 99 artigos. Deste montante, fez-se a leitura de todos e filtraram-se os artigos que verdadeiramente estavam inseridos na área de interesse da pesquisa, reduzindo a amostra para 49 artigos, apresentados na Tabela 1.

Tabela 1. Pesquisa por combinação de palavras-chave.

\begin{tabular}{ccc}
\hline Palavra-Chave 1 & Palavra-Chave 2 & $\begin{array}{c}\text { Número de artigos } \\
\text { encontrados }\end{array}$ \\
\hline Photovoltaic Energy & Performance assessment & 14 \\
Photovoltaic Energy & Performance indicators & 17 \\
Photovoltaic Energy & Multi-criteria decision & 18 \\
\hline
\end{tabular}

Fonte: Science Direct, Scopus, CAPES e Emerald (2016)

Para conduzir o estudo fez-se a análise dos 49 artigos resultantes das combinações de palavras-chave, deste total, durante o período de publicação pesquisado, compreendido entre os anos de 2011 e 2016, observou-se um crescimento no número de artigos publicados, partindo de três artigos do ano de 2011 para 19 artigos no ano de 2015, e um total de seis no primeiro semestre de 2016. Sendo assim, percebe-se o aumento do interesse de pesquisadores na área de gestão de energia fotovoltaica.

\section{Resultados e discussões}

\subsection{Países Pesquisadores e Relacionamentos Colaborativos}

A análise da frequência de artigos publicados em relação à origem do primeiro autor foi descrita em forma gráfica, conforme a Figura 2. A origem do primeiro autor é identificada pelo país em que se localiza a instituição afiliada. Sendo assim, no ranking dos 11 países mais produtivos encontram-se a Itália em primeiro lugar, a Espanha em segundo e a Suíça em terceiro. Além disso, é importante destacar o fato de que dos 11 países citados no ranking, 7 pertencem ao continente Europeu. Esse dado vai ao encontro de pesquisas que apontam a União Europeia (UE) como uma das estrelas para um mundo mais ecológico e sustentável. Além disso, os painéis solares fotovoltaicos (PV) são uma parte essencial da estratégia de aumentar para $20 \%$ a quota de energias renováveis na produção total de energia na Europa até 2020 [7]. Esse mesmo estudo afirma que a Alemanha tem, com larga vantagem, o maior mercado em termos de capacidade instalada total na UE, seguida pela Itália, Espanha e França. Destes países, 
a Alemanha foi o único que não apareceu na frequência de pesquisas.

Ao relacionar esses dados com a pesquisa realizada por Huibin Du, aponta-se os Estados Unidos como o país de mais alta produtividade de artigos publicados no âmbito geral de energia fotovoltaica, produzindo mais que o dobro do segundo colocado [8]. Percebe-se que este país se apresenta com um pequeno número de artigos, o que demonstra a lacuna de pesquisa de uma grande potência que provavelmente mantém apenas o foco no estudo técnico da geração e distribuição de energia fotovoltaica e não um estudo que compreenda as áreas de Engenharia de Produção e Administração.

Ao preocupar-se em pesquisar a gestão da energia fotovoltaica, esses países podem vir a tornarem-se mais competitivos em comparação àqueles que concentram seus esforços apenas no desenvolvimento da tecnologia de energia fotovoltaica.

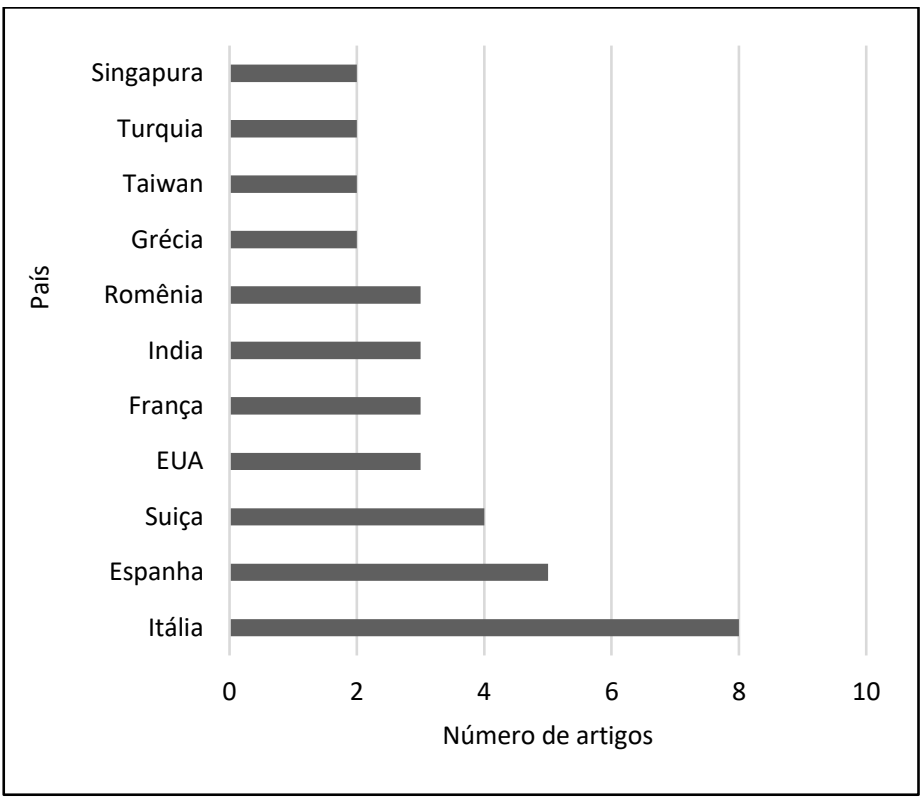

Figura 2 - Países mais produtivos.

Além da análise do país em que se encontra a instituição afiliada do primeiro autor, foi construída a relação de colaboração acadêmica entre os países, conforme a Figura 3. Essa relação foi construída através da rede de conexões, na qual o nó representa o país e as linhas a intensidade de relacionamento entre os países dos pesquisadores. Neste caso, não houve nenhum país que se relacionou em mais de um artigo com um país específico, por isso as linhas tem a mesma espessura, que significam 1 (um) artigo.

Destaca-se a participação da Áustria na rede de cooperação. O país apresentou o maior número de relações acadêmicas, mesmo não fazendo parte no ranking da Figura 2. Dessa forma, percebe-se a tendência de cooperação da Áustria em pesquisas desenvolvidas na área, colaborando com Eslovênia, Alemanha e Bélgica [9], e com Omã e Malásia [10]. Nestes parâmetros, a Áustria vem seguida da Bélgica, que apresentou relacionamento com quatro países, conforme o estudo de $[9,11]$. A partir da leitura e análise das pesquisas, verificou-se que a Alemanha, Espanha, Itália e França apresentaram relacionamento com três países em suas publicações [9, 12-14].

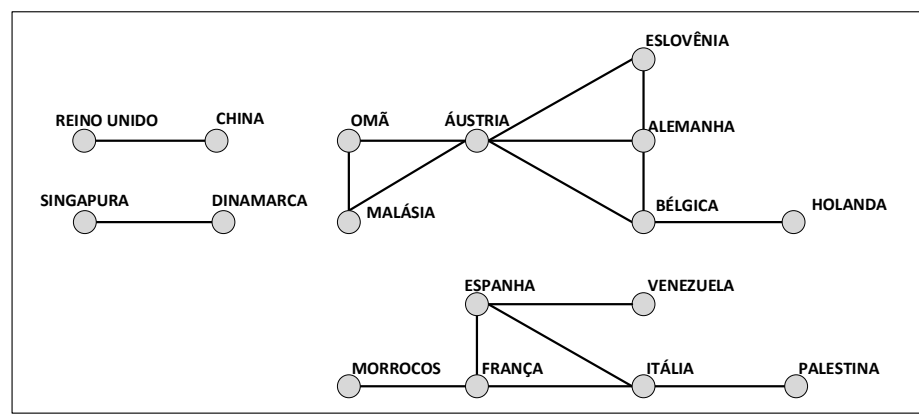

Figura 3 - As relações colaborativas acadêmicas entre os países (redes de cooperação).

É interessante observar que alguns estudos sobre a energia fotovoltaica possuem conexão entre países de localidades próximas, como os países europeus, onde as características geográficas e solarimétricas são semelhantes. No entanto, são verificadas relações acadêmicas entre diferentes continentes, como o relacionamento da Áustria com os países Omã e Malásia [10]. Nesta pesquisa é avaliado o desempenho e a viabilidade de um sistema de energia fotovoltaica conectado a uma rede de 1,4 kW sob condições climatéricas desérticas, o que aponta a troca de conhecimento entre a Áustria, com sua notoriedade em desenvolvimento de pesquisas, e as necessidades dos países com essas condições climáticas. Analisa-se então, que a cooperação entre os diferentes países apoia o desenvolvimento da gestão dessa tecnologia, a partir do momento em que é possível realizar testes e simulações em diferentes zonas climáticas e políticas.

\section{2 Áreas foco da pesquisa}

Em relação ao escopo dos artigos estudados, além das palavras-chave que filtraram a pesquisa para os 49 artigos, foi investigado quais são as grandes áreas que estes englobam. Para isso, realizou-se a leitura completa de cada artigo para identificar qual a grande área em que o mesmo se enquadrava prioritariamente. As áreas são apresentadas na Figura 4.

Como previsto, a grande área da Engenharia Elétrica ainda é o foco da maior parte dos artigos que tratam da energia fotovoltaica, o que demonstra a carência de pesquisas de outras grandes áreas que envolvem este assunto, admitindo lacunas pertinentes para pesquisadores. A exemplo disso há a grande área da Arquitetura, na gestão de projetos de design e aplicação em ambientes públicos e privados, bem como a grande área da Engenharia Mecânica, como gestão de produção de estruturas de fixação e desenvolvimento de novos produtos. Pode-se citar ainda 
a Engenharia Química, como gestão da fabricação de células de silício e as áreas da Economia, Administração e Engenharia de Produção, com estudos para implementação de técnicas e ferramentas de gestão em usinas fotovoltaicas.

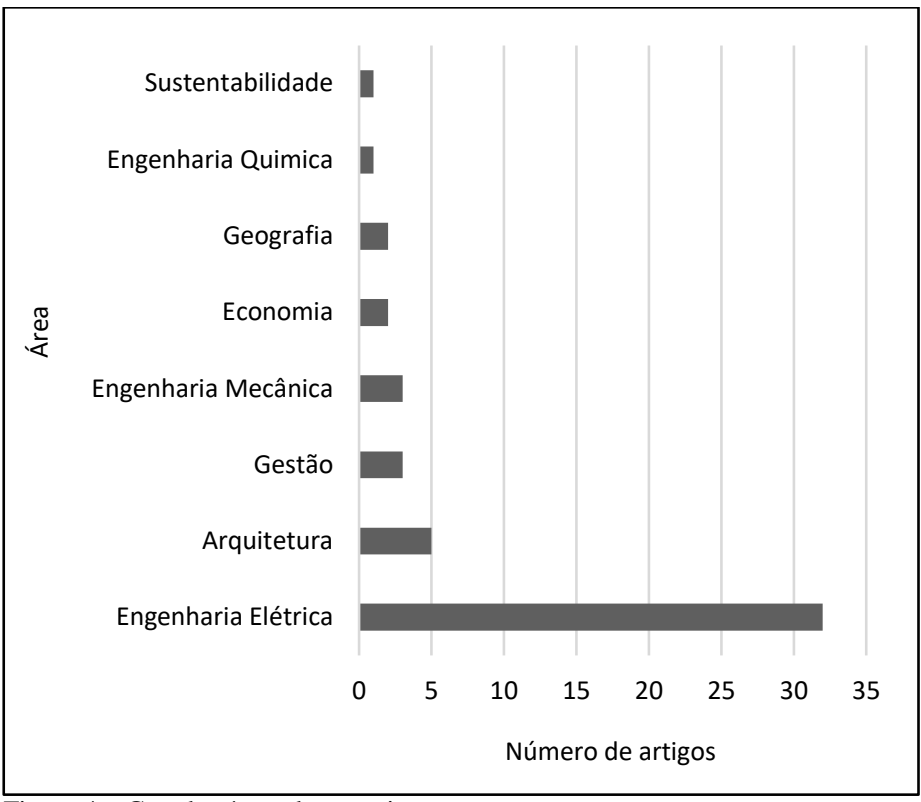

Figura 4 - Grandes áreas de pesquisa.

É importante ressaltar que apenas 3 dos 49 artigos realmente abordaram ferramentas de gestão na geração de energia fotovoltaica, destes três, duas pesquisas tratam da escolha entre fontes de geração de energia alternativa através da utilização da ferramenta e metodologia Analytic Hierarchy Process (AHP) e Multi-criteria Decision [15-16]. Outro estudo analisou a política de inovação utilizando a ferramenta Multi-criteria Decision [17]. A ferramenta Analytic Hierarchy Process (AHP) surgiu para modelar problemas complexos de gestão e problemas específicos com aplicações e técnicas de tomada de decisão multicritério que sejam capazes de auxiliar os gestores [18].

Sequencialmente, foi realizada a análise das palavraschave dos artigos pesquisados. O ranking da frequência das palavras pode ser visualizado na Figura 5. Sem modificações na forma escrita, foram consideradas apenas as palavras exatamente iguais àquelas existentes nos artigos.

Em decorrência de a pesquisa consistir em buscar artigos com as palavras-chave "Performance assessment", "Performance indicators" e "Multi-critéria decision" combinadas com "Photovoltaic Energy", tem-se como maior frequência palavras relacionadas a estas. Pode-se destacar a frequência de pesquisas que envolvem "Geographic Information Systems" (GIS), um sistema para analisar e gerenciar dados espaciais ou geográficos. Nestes casos, essa metodologia foi aplicada em 4 artigos [16, 1921]. Verificou-se que a metodologia de Análise Multicritério de Apoio à Tomada de Decisão é utilizada por estes pesquisadores para compreender os aspectos geográficos, e então determinar sistematicamente a melhor localização para a construção de usinas solares através da análise dos multicritérios identificados e ponderados.

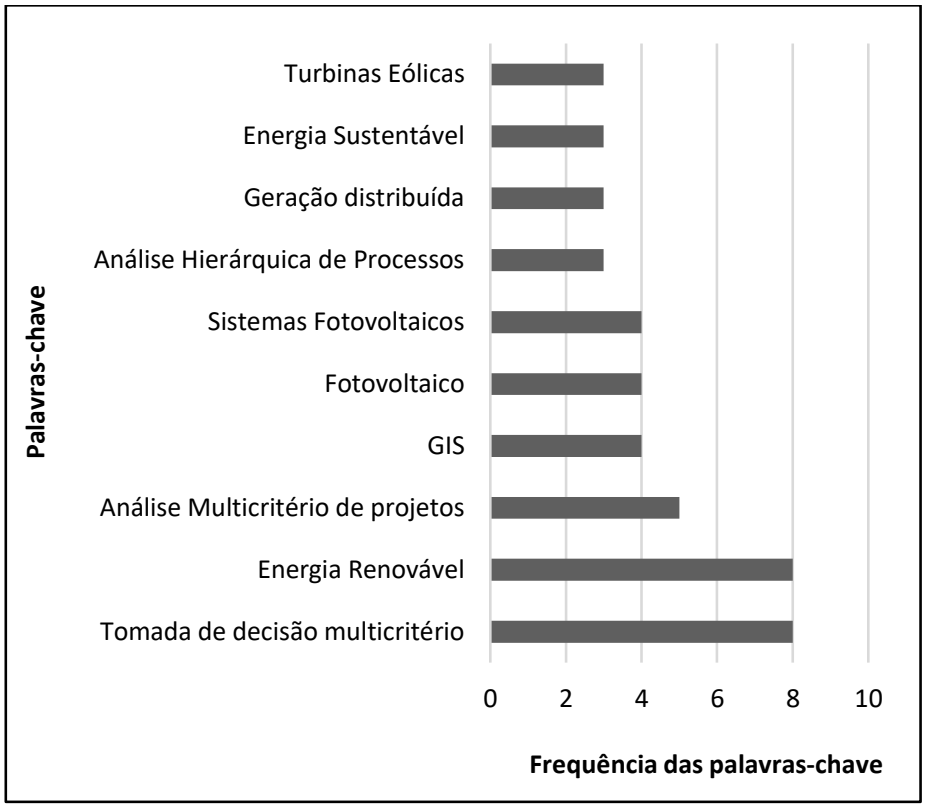

Figura 5 - Ranking de frequência de palavras.

Além disso, é importante ressaltar que muitos estudos se baseiam no comparativo de diferentes energias alternativas, evidenciando as palavras-chaves "Renewable energy" e "Sustainable energy", sendo a energia solar a mais comumente comparada a "Wind". Nessas comparações são utilizadas técnicas de Análise Hierárquica de Processo, na qual os critérios são avaliados em relação às fontes alternativas de energia.

\subsection{Impacto das publicações}

No campo do impacto das publicações selecionadas, prioritariamente, foi realizada a análise dos seus meios de publicação. A partir disso encontrou-se uma frequência maior que 1 em apenas oito periódicos. Estes oito periódicos publicaram 30 dos 49 artigos pesquisados. A Figura 6 apresenta o gráfico do ranking dos 8 periódicos que mais publicaram artigos e o fator de impacto de cada um.

Dentre o total de artigos, dez foram publicados no periódico "Renewable and Sustainable Energy Reviews", que possui um fator de impacto de 6,798. O periódico com maior fator de impacto identificado na pesquisa foi o "Progress in Photovoltaics: Research and Applications" com 7,365, o qual contempla dois artigos publicados com abrangência dos termos selecionados neste estudo. $\mathrm{O}$ restante dos artigos possui um fator de impacto na faixa de 3,00 a 5,00. Dessa forma, foi constatado que as pesquisas que englobam essa área possuem alto fator de impacto para a comunidade acadêmica, essa observação pode ser 
apoiada ao fato de a presença da energia fotovoltaica consolidarse cada vez mais no mundo e a identificação de indicadores e ferramentas que auxiliem os gestores mostrar-se como uma necessidade inerente no avanço da competitividade deste setor.

Dando sequência às análises, verificou-se o número de citações dos artigos na plataforma Google Scholar. O total de citações dos 49 artigos é de 676, sendo que apenas 6 não possuem citações, e $50 \%$ dos artigos possuem mais que 10 citações. A Tabela 2 apresenta o ranking dos 10 artigos mais citados.

$\mathrm{O}$ artigo mais citado, do pesquisador Sanchez-Lozano [19], possui 79 citações contabilizadas pelo Google Scholar, e trata de uma análise combinada de GIS e Multi-criteria Decision para analisar quais os locais mais apropriados para explorar a produção de energia solar no sudeste da Espanha, baseado em critérios pré- selecionados pelos autores. Seguido deste, o segundo artigo mais citado, do pesquisador Sharma [22], possui 50 citações, o qual estuda a avaliação de desempenho de diferentes tecnologias de energia solar fotovoltaica, comparadas em ambiente de condições similares, realizado na Índia.

Dentre esses 10 artigos mais citados em comparação com o montante selecionado, 6 fazem parte da grande área de Engenharia Elétrica, e os 4 restantes referem-se cada um a uma diferente grande área, sendo elas: Engenharia Mecânica, Engenharia Química, Economia e Geografia. Dessa forma, evidencia-se que as pesquisas conduzidas por diferentes grandes áreas, além da Engenharia Elétrica, também apresentam pesquisas de grande impacto, o que identifica o setor de energia solar como sendo multidisciplinar.

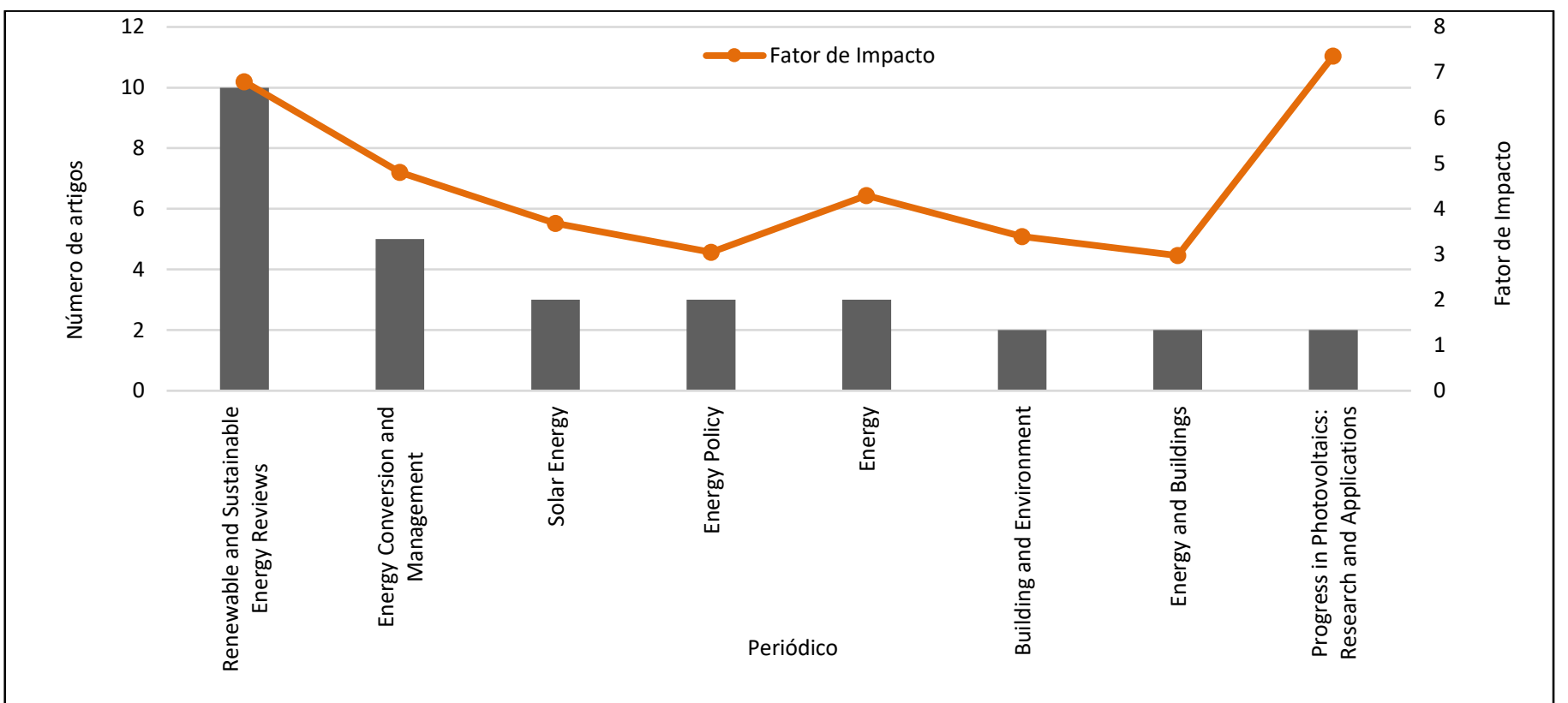

Figura 6 - Ranking dos periódicos

\section{Considerações Finais}

$\mathrm{O}$ estudo buscou analisar as características da literatura sobre energia fotovoltaica, utilizando a palavra-chave "Photovoltaic Energy", em cruzamento com as palavras-chave "Performance Assessment", "Performance Indicators" e "Multicriteria decisions", entre artigos acadêmicos publicados ao longo dos anos 2011 e 2016, contemplando uma análise dos últimos 5 anos. Através da implementação dessa metodologia, obteve-se um retorno de 99 artigos. Deste montante, fez-se a leitura do total dos textos e filtraram-se os artigos que verdadeiramente estavam inseridos na área de pesquisa de energia fotovoltaica, reduzindo a amostra para 49 artigos. A leitura destes artigos confirmou o potencial de pesquisas internacionais envolvendo a temática da energia fotovoltaica e os benefícios desta energia renovável, confirmando que as pesquisas abordadas possuem um alto grau de impacto e de citações, envolvendo a relação entre diversos países. Porém, os dados coletados, demonstram um baixo índice de pesquisas desenvolvidas na área de gestão da energia, pois apenas 3 dos 49 artigos realmente fazem o uso de ferramentas de apoio à tomada de decisão. Além disso, não são encontrados estudos que se utilizem dos conceitos estudados com o intuito de gerir a energia fotovoltaica a partir das técnicas de decisões utilizando a análise multicritério e estudos que apresentem uma preocupação no desenvolvimento de sistemas de mensuração de desempenho ou de desenvolvimento de indicadores de desempenho para as unidades geradoras de energia fotovoltaica.

Sendo assim, por não terem sido encontrados estudos que se preocupam em desenvolver ou aplicar formas de avaliação de desempenho e indicadores de desempenho para a geração de 
energia fotovoltaica, muitas geradoras de energia podem deixar de avançar na manutenção, investimento em novas tecnologias e na competitividade do setor. Para que o crescimento da geração de energia fotovoltaica continue em crescimento exponencial é importante que os investidores e gestores estejam munidos de informações acerca de seu desempenho, e consequentemente, sua competitividade perante outras fontes de energia.

Tabela 2. Ranking dos 10 artigos mais citados

\begin{tabular}{|c|c|c|c|c|c|}
\hline Citações & Ano & Título & Autores & Instituição & País \\
\hline 79 & 2013 & $\begin{array}{c}\text { Geographical Information Systems (GIS) and Multi- } \\
\text { Criteria Decision Making (MCDM) methods for the } \\
\text { evaluation of solar farms locations: Case study in } \\
\text { south-eastern Spain }\end{array}$ & $\begin{array}{c}\text { Juan M.Sánchez-Lozano, } \\
\text { JerónimoTeruel-Solano, } \\
\text { PedroL.Soto-Elvira, e M. Socorro } \\
\text { García-Cascales. }\end{array}$ & $\begin{array}{l}\text { Universidad Politécnica de } \\
\text { Cartagena }\end{array}$ & Espanha \\
\hline 50 & 2013 & $\begin{array}{l}\text { Performance assessment of different solar photovoltaic } \\
\text { technologies under similar outdoor conditions }\end{array}$ & $\begin{array}{l}\text { Vikrant Sharma, Arun Kumar, O.S. } \\
\text { Sastry, S.S. Chandel. }\end{array}$ & $\begin{array}{l}\text { Centre for Energy and } \\
\text { Environment National } \\
\text { Institute of Technology }\end{array}$ & India \\
\hline 41 & 2011 & $\begin{array}{l}\text { Multicriteria selection aiding related to photovoltaic } \\
\text { plants on farming fields on Corsicais4land: A real case } \\
\text { study using the ELECTRE out ranking framework }\end{array}$ & P. Haurant, P.Oberti, M.Muselli. & $\begin{array}{l}\text { Université de Corse } \\
\text { Pasquale Paoli }\end{array}$ & França \\
\hline 34 & 2012 & $\begin{array}{l}\text { Evaluation of photovoltaic cells in a multi-criteria } \\
\text { decision making process }\end{array}$ & $\begin{array}{l}\text { M. Socorro García-Cascales, M. } \\
\text { Teresa Lamata, J. Miguel Sánchez- } \\
\text { Lozano }\end{array}$ & $\begin{array}{l}\text { Universidad Politécnica de } \\
\text { Cartagena }\end{array}$ & Espanha \\
\hline 28 & 2014 & $\begin{array}{l}\text { Design of hybrid power generation systems based on } \\
\text { multi criteria decision analysis }\end{array}$ & $\begin{array}{l}\text { M. Alsayed, M. Cacciato, G. } \\
\text { Scarcella, G. Scelba. }\end{array}$ & $\begin{array}{l}\text { An-Najah National } \\
\text { University }\end{array}$ & Palestina \\
\hline 28 & 2014 & $\begin{array}{l}\text { Unsaturated flow modeling in performance } \\
\text { assessments forth e Yucca Mountain disposal system } \\
\text { for spent nuclear fuelandhigh-level radioactive waste }\end{array}$ & $\begin{array}{l}\text { Rob P. Rechard, Jens T. Birkholzer, } \\
\text { Yu-Shu Wu, Joshua S.Stein, James E. } \\
\text { Houseworth. }\end{array}$ & $\begin{array}{l}\text { Sandia National } \\
\text { Laboratories }\end{array}$ & EUA \\
\hline 27 & 2015 & $\begin{array}{c}\text { Don't just follow the sun - A global assessment of } \\
\text { economic performance for residential building } \\
\text { photovoltaics }\end{array}$ & $\begin{array}{c}\text { Tillmann Lang, Erik Gloerfeld, } \\
\text { Bastien Girod }\end{array}$ & $\begin{array}{l}\text { Swiss Federal Institute of } \\
\text { Technology Zurich }\end{array}$ & Suiça \\
\hline 26 & 2014 & $\begin{array}{c}\text { Lifetime performance of semi-transparent building- } \\
\text { integrated photovoltaic (BIPV) glazing systems in the } \\
\text { tropics }\end{array}$ & Poh Khai Ng, Nalanie Mithraratne & $\begin{array}{l}\text { National University of } \\
\text { Singapore }\end{array}$ & Singapura \\
\hline 24 & 2013 & $\begin{array}{l}\text { Lifecycle performance assessment of fuel cell/battery } \\
\text { electric vehicles }\end{array}$ & $\begin{array}{l}\text { Jenn-Jiang Hwang, Jenn-Kun Kuo, } \\
\text { Wei Wub, Wei-Ru Chang, Chih- } \\
\text { Hong Lin, Song-En Wanga }\end{array}$ & $\begin{array}{l}\text { National University of } \\
\text { Tainan }\end{array}$ & Taiwan \\
\hline 23 & 2015 & $\begin{array}{l}\text { Concentrated photovoltaic thermal(CPVT) solar } \\
\text { collector systems: Part II - Implemented systems, } \\
\text { performance assessment, and future directions }\end{array}$ & Omar Z. Sharaf, Mehmet F. Orhan & $\begin{array}{l}\text { American University of } \\
\text { Sharjah }\end{array}$ & $\begin{array}{c}\text { Emirados } \\
\text { Árabes } \\
\text { Unidos }\end{array}$ \\
\hline
\end{tabular}

\section{Agradecimentos}

Os autores agradecem ao INCTGD, CAPES, CNPq e FAPERGS pelo apoio financeiro recebido para o desenvolvimento deste trabalho. Michels e Siluk foram apoiados por uma bolsa de pesquisa do $\mathrm{CNPq}$ - Brasil. O presente trabalho foi realizado com o apoio do INCTGD e das agências financiadoras (processo CNPq 465640 / 2014-1, processo CAPES no 23038.000776 / 2017-54 e FAPERGS 17 / 2551-0000517-1).

\section{THE MANAGEMENT OF PHOTOVOLTAIC SOLAR ENERGY FROM THE PERSPECTIVE OF BIBLIOMETRICS}

ABSTRACT: Photovoltaic solar power generation is growing exponentially around the world, and this technical and commercial growth must be supported by energy management studies through decision support tools. In this sense, this article investigated, from a bibliometric analysis, the current scenario of academic research in the Photovoltaic Solar Energy area, in conjunction with the concepts of Performance Evaluation, Performance Indicators and Multicriteria Decision Analysis, over a period of 5 Years from 2011 to 2016. Through this search, 49 articles were found and the international potential of photovoltaic energy studies was confirmed, confirming that the researchers studies have a high degree of impact and citations involving several countries. However, no studies were found. This confirms a gap in the scientific research environment that must be fulfilled, as studies in this area are essential to ensure the continued growth of photovoltaic solar energy in the world.

Keywords: Photovoltaic Energy. Energy Management. Bibliometric. 


\section{Referências}

[1] LI, X; HUI, D; LAI, X. Battery Energy Storage Station (BESS)-Based Smoothing Control of Photovoltaic (PV) and Wind Power Generation Fluctuations. IEEE Transactions on Sustainable Energy, Vol. 4, n. 2, 2013.

[2] DING, M; XU, Z. An empirical model for capacity credit evaluation of utilityscale PV plant. IEEE Transactions on Sustainable Energy, Vol. 8, n. 1, 2016.

[3] ABAEI, M.M. et al.. Developing a novel risk-based methodology for multicriteria decision making in marine renewable energy applications. Renewable Energy, Vol. 102, p.341-348, 2016.

[4] BEHZADIAN, M. et al. A comprehensive literature review on methodologies and applications. European Journal of Operational Research, Vol. 200, n. 1, p. 198$215,2009$.

[5] TSAY M-Y. A bibliometric analysis of hydrogen energy literature, $1965 \mathrm{e}$ 2005. Scientometrics. Vol 75, n. 3, p. 421-438, 2008

[6] ZUPIC, T. ČATER. Bibliometric methods in management and organization Organizational Research Methods, Vol. 18, p. 429-472, 2015.

[7] L. DE BOECK, S. et al. Comparison of support policies for residential photovoltaic systems in the major EU markets through investment profitability. Renewable Energy. Vol. 87, p. 42-53, 2016.

[8] DU, H. et al. A bibliographic analysis of recent solar energy literatures: The expansion and evolution of a research field. Renewable Energy, Vol. 66, p. 696706,2014

[9] BLETTERIE, B. et al. Development of innovative voltage control for distribution networkswith high photovoltaic penetration. Progress in photovoltaics. Research and applications, Vol. 22, p. 747-759, 2012.

[10] KAZEM, H. A. et al. Performance and feasibility assessment of a $1.4 \mathrm{~kW}$ roof topgrid-connected photovoltaic power system under desertic weather conditions. Energy and Buildings, Vol. 82, p. 123-129, 2014

[11] CLAESSEN, F. N. et al. A. Comparative analysis of tertiary control systems for smart grids using the Flex Street model. Renewable Energy, Vol. 69, p. 260270,2014

[12] KUZNETSOVA, E.; RUIZ, C.; LI, Y. F.; ZIO, E. Analysis of robust optimization for decentralized microgrid energy management under uncertainty. Electrical Power and Energy Systemns, Vol. 64, p. 815-832, 2015.

[13] ROJAS-ZERPA, J. C.; YUSTA, J. M. Application of multicriteria decision methods for electric supply planning in rural and remote areas. Renewable and Sustainable Energy Reviews, Vol 52, p. 557-571, 2015.

[14] ALSAYED, M.et al. Design of hybrid power generation systems based on multi criteria decision analysis. Solar Energy, Vol 105, p. 548-560, 2014.

[15] MA, D.; CHANG, C. C.; HUNG, S. W. The selection of technology for latestarters: A case study of the energy-smart photovoltaic industry. Economic Modelling. Vol. 35, p. 10-20, 2013.

[16] TAHRI, M.; HAKDAOUI, M; MAANAN, M. The evaluation of solar farm locations applying Geographic Information System and Multi-Criteria DecisionMaking methods: Case study in southern Morocco. Renewable and Sustainable Energy Reviews. Vol. 51,p. 1354-1362, 2015.
[17] SARUCAN, A. et al. Hierarchy Grey Relational Analysis for Selecting the Renewable Electricity Generation Technologies. Proceedings of the World Congress on Engineering, Vol II WCE 2011, July 6 - 8, 2011, London, U.K, 2011.

[18] MIEZA, I, Z.; YEPES, V.; MORENO-JIMENEZ, J. M. A systematic review of application of multi-criteria decision analysis for aging-dam management. Journal of Cleaner Production. Vol. 147, p. 217- 230. 2017.

[19] SÁNCHEZ-LOZANO, J. M. et al. Geographical Information Systems (GIS) and Multi-Criteria Decision Making (MCDM) methods for thee valuation of solar farms locations: Case study in south-eastern Spain. Renewable and Sustainable Energy Reviews, Vol 24, p. 544-556, 2013.

[20] GOE, M.; GAUSTAD, G.; TOMASZEWSKI. System tradeoffs in siting a solar photovoltaic material recovery infrastructure. Journal of Environmental Management, Vol 160, p. 154-166, 2015.

[21] ROA-ESCALANTE, G. J. et al. The effects of photovoltaic electricity injection into microgrids: Combination of Geographical Information Systems, multicriteria decision methods and electronic control modeling. Energy Conversion and Management, Vol. 96, p. 89-99, 2015.

[22] SHARMA, V. et al. Performance assessment of different solar photovoltaic technologies under similar outdoor conditions. Energy, Vol 58, p. 511-518, 2013. 